\title{
Über die Auswahl der Indikatoren für die acidimetrische Bestimmung der Borsäure.
}

\author{
Von \\ Dr. Johannes Prescher. \\ Mitteilung a dem Statlichen Chemischen Untersuchungsamte für die \\ Auslandsfleischbeschau in Cleve (Vorsteher: Dr. M. Fritzsche).
}

[Eingegangen am 14. November 1918.]

Obwohl nach den günstigen Erfahrunger, die Beythien und Hempel ${ }^{1}$, Lührig${ }^{2}$ ), K. Windiseh${ }^{3}$ ), B. Fischer ${ }^{4}$, Hönig und Spitz ${ }^{5}$, Joergensen ${ }^{6}$ ) u. a. ${ }^{7}$ mit der Verwendung von Phenolphthalein als alleinigem Indicator bei der titrimetrischen Borsäurebestimmung unter Zusatz mehrwertiger Alkohole (Glycerin, Mannit) gemacht hatten, anzunehnien war, daß die Ergebnisse genannter Forscher den Praktiker vollauf befriedigen würden, ist neuerdings von $\mathrm{John}^{8}$ ) Methylrot an Stelle von Methylorange empfohlen worden, weil hiermit der Umschlag bei Gegenwart von Carbonat deutlicher sei, als mit dem Methylorangeindicator.

Letzterem gegenüber ist Borsäure als schwache Säure ${ }^{9}$ ) ebenso wie Blausäure, Kohlensäure, Schwefelwasserstoff, arsenige Säure u. a. in wässeriger Lösung völlig indifferent; sie bleibt es auch nach der Verstärkung mit neutralisiertem Glycerin und kann in diesem Falle erst nach Zugabe eines Indicators (Phenolphthalein) gemessen werden, der den wahren Endpunkt der Sättigung mit Alkali deutlich zu erkennen gestattet. - Da die Eliminierung der Borsäure aus alkalischen Aschen erst nach der Übersättigung mit stärkerer Säure (Salzsäure) erfolgen kann, aus phosphathaltigen A schen alsdann Phosphorsäure hinzutritt, so hat die Ausschaltung aller fremden Säuren der Titration der gleichzeitig freigewordenen Borsäure voranzugehen. Zur Erkennung der eingetretenen Neutralisation in Gegenwart von Borsäure sind sowohl Methylorange wie Phenolphthalein gebräuchlich, ersteres, weil bei dessen ausgesprochenem Säure-

1) Diese Zeitschrift 1899, 2, 842; 1902, 5, 764; 1905, 10, 283.

\&) Pharm. Zentralh. 1901, 42, 50.

a) Diese Zeitschrift 1905, 11, 659.

*) Diese Zeitschrift $1900,3,17$.

5) Zeitschr. angew. Chem. 1896, 9, 549.

в) Nordisk farmaceutisk Tidskrift 1895, 213; Zeitschr. angew. Chemie 1897, 10, 5.

7) Arch. Pharm. 1904, 242, 194.

8) Süddeutsche Apothekerzeitung 1917, 520 u. 372; Apothekerzeitung 1917, 32, 502; Pharmaz. Zentralh. 1917, 58, 618; Chem. Zentralbl. 1917, I, 1141.

9) In dem Borylphosphat $\mathrm{PO}_{\ddagger} \mathrm{B}$ spielt sie sogar die Rolle efner dreisäurigen Base. Ich verweise hier gleichzeitig auf das eigenartige Verhalten einer mit Glycerin sauer ge-


durch Phenolphthalein geröteten Glycerinlösung und einer mit Phenolphthalein gefärbten Bo raxlösung. Während in ersterem Falle eine alkalische Flüssigkeit entsteht, tritt im zweiten durch Vermischung $\mathrm{zweier}$ alkalischer Lösungen eine stark sauere Flüssigkeit auf. (Vergl. Hundeshagen, Zeitschr. anorgan. Chem. III, 1901). Merkwürdig ist auch das heftige Aulbrausen beim Mischen von Borax- und Sodalösung mit Glycerin. (Vergl. D. Klein, Compt. rend. 1878, 46, 826 oder J. Prescher, Die praktisehen Methoden der Bestimmung der Borsäure, Lübeck 1905, S. 27, Verlag von Charles Coleman.) 
charakter ihm gegenüber Borsäure sich neutral verhält, letzteres, weil bei Zugabe dieses empfindlichsten aller Säurereagenzien die schwache Borsäure zwar an sich bereits eine kleine Menge Alkali verbraucht, jedoch in so unvollkommener und auf das Endergebnis unter Berücksichtigung bestimmter Titer ohne nennenswerten Einfluß bleibender Weise, daß der Vorteil der Verwendung von Phenolphthalein als alleinigem Indicator in die Augen springen muß, wenn man folgendes berücksichtigt:

Bei Anwendung von Methylorange tritt beim Neutralisieren freier Phosphorsäure Unschlag von Rot in Gelb bereits ein, wenn das erste Molekül Natriumhydroxyd über den Punkt der Verwandlung von Phosphorsäure in Dinatriumphosphat hinaus verbraucht oder $1 / 3$ der Phosphorsäure neutralisiert ist:

$$
\mathrm{PO}_{4} \mathrm{H}_{3}+\mathrm{NaOH}=\mathrm{PO}_{4} \mathrm{H}_{2} \mathrm{Na}+\mathrm{H}_{2} \mathrm{O} \text {. }
$$

Das primäre Natriumphosphat verhält sich somit gegenüber Methylorange neutral. Versetzt man hirgegen Phosphorsäure mit Phenolphthalein und titriert mit normaler Lauge, so tritt bleibende Rötung erst dann ein, wenn ${ }^{2} / 3$ der Phosphorsäure neutralisiert sind:

$$
\mathrm{PO}_{4} \mathrm{H}_{3}+2 \mathrm{NaOH}=\mathrm{PO}_{4} \mathrm{HNa}_{2}+2 \mathrm{H}_{2} \mathrm{O} \text {. }
$$

Phosphorsäure verbraucht in diesem Falle also doppelt soviel Alkali wie in ersterem; sie ist daher zu entfernen ${ }^{1}$ ), sollen beim Weitertitrieren der durch Methylorange als beendet angezeigten. Neutralisation der Säuren für die nach Zugabe von Glycerin gebildete Glycerinborsäure $\left(\mathrm{C}_{3} \mathrm{H}_{5} \mathrm{O}_{2} \mathrm{OH} . \mathrm{BOH}\right)$ in Gegenwart von Phenolphıhalein genügend genaue Werte angezeigt und der Mehrvierbrauch von Alkali fälschlicherweise nicht als Borsäure in Rechnung gesetzt werden. Jedes in Lösung befindliche Dezigramm Phosphorsäure erfordert $2,04 \mathrm{ccm} \mathrm{1/2} \mathrm{N.-Lauge,} \mathrm{entsprechend}$ einem Fehler von $0,063 \mathrm{~g}$ Borsäure.

Es muß daber einleuchten, die glatte Titration in der Weice durchzuführen, dab man von vornherein, also auch für die erste Einstellung denselben Indicator wählt, wenn hierdurch von der Abscheidung der Phosphorsäure, was beim Phenolphthalein der Fall ist, Abstand genommen werden kann. Eins ist indes hierbei zu berïcksichtigen. Der Gleichung

$$
\mathrm{BO}_{3} \mathrm{H}_{3}+\mathrm{NaOH}=\mathrm{BO}_{2} \mathrm{Na}+2 \mathrm{H}_{2} \mathrm{O}
$$

entsprechend, verbrauchen $0,1 \mathrm{~g}$ Borsäure $16,13 \mathrm{~cm} 1 / 10$ N.-Lauge, denn $0,0062 \times 16,13$ $=0,10006 \mathrm{~g}$ Borsäure. Dieser theoretisch berechnete Wert ist aber um ein geringes zu erböhen, wenn der Neutralisationspunkt bis zur ersten Einstellung anstatt mit Methylorange durch Pbenolphthalein ermittelt worden ist; denn dann wurde, da sich Borsäure gegenüber Phenolphthalein sauer verhält, vor dem Glycerinzusatz etwas zu weit titriert. Für $0,1 \mathrm{~g}$ Borsäure in $50 \mathrm{ccm}$ Lösung und Gegenwart von $25 \mathrm{ccm}$ Glycerin hat Beythie ${ }^{2}$ ) die Annahme des Titers 63,29 mg empfohlen, da unter diesen Verhältnissen nur $15,80 \mathrm{ccm} 1 / 10$ N.-Lauge verbraucht werden. Für andere Borsäuremengen und wechselnde Konzentrationen wird der Faktor weiterhin beeinflußt und beträgt bei Anwesenheit von

1) Polenske (Arbeiten a. d. Kaiserl. Gesundheitsamte 1900, 17, 561) versetzt zu diesem Zwecke die filtrierten mit heißem Wasser ausgelaugten Aschenauszüge bis zur sauren Realtion mit Salzäure and scheidet die Phosphorsäure mit Eisenebloid unter nachfolgender Zugabe von Natriumacetat als Ferriphosphat ab, das überschüssige Eisen entfernt er durch Natronlauge.

2) Diese Zeitschrift 1905, 10, 283. 


$$
\begin{aligned}
& 0,1558 \mathrm{~g} \text { Borsäure } 64,39 \mathrm{mg} \mathrm{H}_{3} \mathrm{BO}_{3} \\
& 0,4000 \mathrm{~g} " 66,67 ", "
\end{aligned}
$$

Je nach der Menge vorhandener Borsäure, für deren annähernde Bestimmung zunächst das Äquivalentgewicht der Borsäure dienen kann, ist jeder Analytiker in der Lage, den genauer zu ermittelnden Wert an Hand der Bey thien schen Aufstellungen festzulegen.

Die als Indicator bei der Titration schwacher Basen, besonders von Alkaloiden gebräuchliche $p$-Dimethylaminoazobenzol-o-carbonsäure, das von John an Stelle von Methylorange für die Borsäuretitration herangezogene Methylrot, hat gegenüber der Sulfosäure des Benzol-azodimethylanilins oder des Natrium- oder des Ammoniumsalzes des Sulfanilsäure-azodimetbylanilins den Vorzug, daß die Umschlagfarbe von Gelb in Carmoisinrot auch in Gegenwart von Carbonat deutlicher ist als der Farbenwechsel bei Methylorange. Methylrot verbält sich gegenüber Borsäure sauer und gegenüber Glycerinborsäure ist der Alkaliverbrauch erheblich, wenn auch zur Sättigung unzureichend.

Unter den Hauptpunkten, auf welche man bei Boreäurebestimmungen nach Jörgensen sein Augenmerk richten muß, hat B. Fischer als ersten die Vertreibung der Kohlensäure durch Erhitzen hervorgehoben. Da Borsäure mit Wasserdämpfen flüchtig ist, hat dies nach Ansäuern der alkalischen Aschen in der Kälte nach Überspülen in einen Erlenmeyer-Kolben durch Erhitzen am Rückflußkühler zu erfolgen. Da die hierdurch kohlensäurefrei gewordene Lösung jetzt ebensogut mit Hilfe von Phenolphthalein titrierbar ist, bedarf es deswegen weder des Methyloranges noch des Methylrots. Letzteres zeigt gegenüber vorhandener Phosphorsäure die gleichen Nachteile wie Methylorange; auch hier ließe sich die störende Zwischenreaktion bei der Bildung neutraler Phosphate nur durch Entfernung der Phosphorsäure beheben. Endlich würde das gleichzeitige Einwirken von Methylrot neben Phenolphthalein auf den Farbenwechsel beim Neutralisieren störend wirken, weshalb auch nach dieser Richtung letzteres als Einzelindicator vorzuziehen ist. - Aus der Unmenge von Indicatoren (bei den Vorschlägen, Borax zur Titerstellung der Säuren zu benutzen, ihn also als Urmaß in die Alkalimetrie einzuführen, da er sich beim Titrieren gegenüber gewissen Indikatoren genau so verhält, wie Natronlauge vom gleichen Natrongehalt ${ }^{1}$ ), kamen in früherer Zeit neben Methylorange Lackmus, $A b$ kochungen von Rotholzspähnen, Helianthin, Tropäolin 00, Orange III Poirrier, Kongorot und viele andere in Frage) sei für Borsäuretitrationen noch des Hämatoxylins und des von $B$ arth $\mathrm{e}^{2}$ ) empfohlenen alkoholischen Auszugs roter, französischer Rosenblätter, der gegen Borsäure unempfindlich ist, durch andere Säuren aber rosa und durch Alkalien grün gefärbt wird ${ }^{3}$ ), Erwähnung getan.

Beim Hämatoxylin beeinflussen die undeutlichen, verwaschenen Farbenumschläge die Endergebnisse, auch teilt es in bezug auf die Anzeige des Neutralisationspunktes gegenwärtiger Phosphoräure das Schicksal mit Metbylorange und Methylrot; zu ebensowenig befriedigenden Ergebnissen ist Beerman ${ }^{4}$ ) bei Anwendung des Barthe' schen Rosenblattauszugs gelangt.

1) Vergl. K. Windisch, Diese Zeitschrift 1905, 9, 652.

$\left.{ }^{2}\right)$ Journ. Pharm, Chim, 1894, [5] 29, 163.

2, Diese Zeitschift 1905, 9, 653 .

4) Dissertation Universität Erlangen 1898. 
Nach diesen Ausführungen glaube ich nachgewiesen zu haben, daß Phenolphthalein als bester Borsäureindicator anzusprechen ist, wenigstens der aktivierten, und daB beim Titrieren der Borsäure mit Alkalien in Gegenwart mehrwertiger Alkohole von der Heranziehung anderer Indicatoren auch schon aus ZweckmäßRigkeitsgründen abgesehen werden sollte ${ }^{1}$ ).

1) Vergl. K. W indisch, Diese Zeitschrift 1905, 9, 659 .

\section{Ersatz von Alkohol durch Methylalkohol und denaturierten} Alkohol.

Von

Dr. J. Prescher.

Mitteilung aus dem Statlichen Chemischen Untersuchungsame far die AusIandsfleischbeschan in Cleve (Vorsteher: Dr. M. Fritzsche).

[Eingegangen am 23. November 1918.]

\section{I. Über Versuche, beim Gottlieb - Röse'schen Verfahren Methylalkohol an Stelle von Äthylalkohol zu verwenden.}

Seiner Leichtlöslichkeit in Alkohol zufolge nimmt Petroläther etwa 1/3 Volumen der anzuwendenden Menge des ersteren in das Röse'sche Fettausschüttelungsgemisch auf, soda月, wie angestellte Versuche ergeben haben, die wässerige, unterhalb der ätherischen liegende Schicht nach Schütteln der jeweilig einander folgenden Mengen Wasser (10 ccm), Ammoniak $\left.{ }^{1}\right)$, Alkohol (10 ccm), Äther $(25 \mathrm{ccm})$, Petroläther $(25 \mathrm{ccm})$ $16,8 \mathrm{ccm}$ beträgt. Bei Verwendung von Methylalkohol (Dichte 0,803 [0,796]) dagegen wird fast die gesamte Alkoholmenge in die wässerige Schicht abgestoßen, indem von der nach dem Mischen von je $10 \mathrm{cem}$ Holzgeist mit $10 \mathrm{ccm} W$ asser $19,5 \mathrm{ccm}$ betragenden Menge $19,3 \mathrm{ccm}$ als wässerige Schicht zurückbleiben. Schüttelt man zunächst nur mit Äther $(25 \mathrm{ccm})$ allein aus, so gehen in diesen ungleich größere Mengen Äthylalkohol über und die wässerige Schicht macht dann nur $13 \mathrm{ccm}$ aus. Metbylalkohol hingegen verhält sich auch Äther gegenüber schon ziemlich passiv, denn von dem ursprünglich auf $19,5 \mathrm{ccm}$ sich belaufenden wässerigen Holzgeistgemische werden nur $0,75 \mathrm{ccm}$ an die ätherische Schicht abgegeben.

Wenn also bei Milchfettbestimmungen vor der Zugabe des Alkohols zur Milch noch die üblichen (1 bis) $2 \mathrm{cem}$ Ammoniak zugesetzt werden, so grenzt die wässerige Schicht bei Anwendung von Methylalkohol etwa bei Strich $21 \mathrm{ab}$, während mit Äthylalkohol in den meisten Fällen die Trennungslinie der wässerigen Schicht zwischen $18,8-19,2 \mathrm{zu}$ liegen kommt.

Bei Fettbestimmungen in Magermilch und Buttermilch, mehr aber noch bei Vollmilch und ganz besonders bei Kakaofettbestimmungen bilden sich bei Verwendung von Methylalkobol unterhalb der ätherischen Zone breitere wolkige Übergänge, die das Ablesen erschweren, wodurch die Endergebnisse mehr oder weniger stark beeinfluft werden. Bei Äthylalkohol hingegen ist die Trennungslinie in allen diesen Fällen eine scharfe, wenn das Gelieren, das bei der Fettbestimmung in Kakao zu-

1) Bei den Vorversucben wurde von der Zagabe des Ammoniaks abgesehen. 